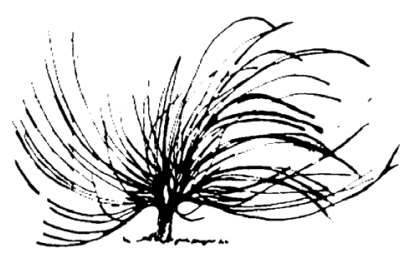

\title{
Pedagogía teatral y anti conductismo, una experiencia de enseñanza y aprendizaje en la comunidad indígena de Amubri ${ }^{1}$
}

\author{
Gerardo Arturo Masis Mata \\ Liceo Rural Aranjuez \\ Costa Rica \\ gera-87-@hotmail.com
}

\begin{abstract}
Resumen
Un sector de la educación actual en Costa Rica se podría comparar con la enfermedad de la "bulimia", en la cual estudiantes memorizan -ingieren- conceptos para luego transcribirlos -regurgitarlos- en una prueba escrita; se genera, con ello, un aprendizaje netamente memorístico y conductual. Lo más peligroso de esto es que se está creando en el estudiantado una "patología educativa". El fomento de la crítica y la motivación por acudir a los espacios áulicos y la significatividad de los aprendizajes ya no tienen campo en un sistema neoliberal, el cual fomenta el dominio de conceptos básicos y mano de obra sumisa. En el presente ensayo, se pretende brindar la herramienta del juego dramático mediante una sistematización de aula en el área de los estudios sociales para socavar la situación del aprendizaje memorístico, utilizando, además,
\end{abstract}

\section{(c) (1) $\Theta \Theta$}

Recibido: 31 de enero de 2020. Aprobado: 2 de setiembre de 2020.

http://dx.doi.org/10.15359/rep.16-1.7

1 Este ensayo corresponde a una versión corregida de una ponencia presentada en el I encuentro Vygotsky y su legado en la investigación en América Latina, celebrado en Costa Rica en setiembre del 2014.

2 Máster en Administración Educativa en la UISIL en el 2019, Licenciado en la Enseñanza de los Estudios Sociales y Educación Cívica en la UNA en el 2016. Docente comprometido con la labor social y aprendizaje contextualizado de las nuevas generaciones. 
como soporte teórico el aprendizaje sociocultural de Lev Vygotsky y la pedagogía teatral, los cuales fomentan aprendizajes contextualizados a la cultura en donde se inserta el estudiantado, en una liberación de la mente y motivación educativa. Todo esto para construir aprendizajes más significativos, no solo para los grupos estudiantiles, sino también para que logren trasmitirlo a las personas que están a su alrededor, y se genere, así, un aprendizaje social, tarea que urge en nuestra sociedad actual.

Palabras clave: Educación, teatro, aprendizaje social, juego dramático, comunidades indígenas, motivación, adoctrinamiento, liberación, paz.

\begin{abstract}
A current sector of the education in Costa Rica could be compared to the disease of "bulimia" in which students memorize -ingest- concepts and then transcribe -regurgitate them- in a written test, thereby generating purely rote and behavioral learning The most dangerous part about this is that an "educational pathology" is being created in the student body. The promotion of criticism and motivation to go to classroom spaces and the significance of learning no longer have room in a neoliberal system, which fosters mastery of basic concepts and submissive labor. This essay aims to propose the tool of dramatic play through a classroom systematization in the area of Social Studies to undermine the situation of rote learning in addition to the use of sociocultural learning of Lev Vygotsky and Theater Pedagogy as theoretical support, which promote learning contextualized in the culture where the student is immersed in a liberation of the mind and educational motivation. This is done to build more meaningful learning not only for students but also for them to transmit it to the people around them, generating social learning, a task that is urgent in our current society.

Keywords: education, theater, social learning, dramatic play, indigenous communities, motivation, indoctrination, liberation, peace.
\end{abstract}




\section{Punto de partida}

\begin{abstract}
$Y$ ahi van los niños,
llenos de sueños, colores e ilusiones se dirigen hacia la maquina domesticadora, donde los cortan, los pintan e introducen un chip y salen como seres perfectos para la sociedad.
\end{abstract}

Original

1 kínder, la escuela y el colegio son instituciones educativas a las cuales se les ha otorgado la formación educativa de niños, niñas y jóvenes; buscan desarrollar destrezas y habilidades para una futura labor; sin embargo, en los últimos años, esta preparación se ha dejado, en gran medida, en manos de estas con la confianza de que van a realizar una muy buena labor. Se ambiciona cubrir grandes unidades de conocimiento acerca de historia, ciencias, matemáticas, entre otras; además, que incluyan valores, respeto, convivencia para que esa niñez y juventud salgan a la calle y puedan vivir plenamente y aportar sustantivamente sus comunidades.

La realidad es otra y hasta se puede decir que está algo alejada de lo que anteriormente se postuló, pues el actual sistema económico y la aplicación de medidas de corte neoliberal en el campo educativo fomentan el tecnicismo y la formación de mano de obra "calificada". Además, la existencia de un déficit en formación educativa y un estado de confort en el que -sin querer generalizar- han caído algunos sujetos educadores, han provocado que en los últimos años la educación venga en declive. Por estas razones se hace fundamental contar con docentes no solamente con compromiso personal con la educación, sino también con preparación académica y pedagógica. A esto, Camargo et al. (2004), aportan:

La formación permanente (o capacitación) del docente debe entenderse como un proceso de actualización que le posibilita realizar su práctica pedagógica y profesional de una manera significativa, pertinente y adecuada a los contextos sociales en que se inscribe y a las poblaciones que atiende. (p. 81)

La formación docente va de la mano de la práctica pedagógica y de la capacitación que se tenga en distintos ámbitos, aspectos idóneos 
para enfrentar la cotidianeidad de las clases y aplicar los conocimientos según el contexto donde se labora.

La generación de docentes de calidad y aprendizajes significativos está ligada a las nuevas teorías educativas; por ello, en el presente ensayo, la sistematización educativa en el área de estudios sociales y educación cívica para décimo y undécimo año se abordan como eje central desde el teatro y su sustento teórico, el aprendizaje sociocultural desarrollado por Vygotsky en 1931. Este mismo pretende no solo desarrollar cualidades escénicas, sino busca contextualizar la educación y asumir roles sociales en los cuales el estudiantado pueda vivir y adaptarse a los retos dentro y fuera de las aulas.

El juego dramático que postula la pedagogía teatral, según García-Huidobro (1996), "es una respuesta educativa para la necesidad de renovar las metodologías que optimizaran el proceso de aprendizaje [en Europa], profundamente alterado por la Segunda Guerra Mundial" (p. 15), la cual tiene la versatilidad de expresarse mediante distintos personajes con una temática eje. Además de personificar acciones, hacer un juego de cambio de roles o imitar a otras personas, es un factor motivante para cualquier estudiante.

El contexto en el cual se desarrolló la presente investigación fue la zona indígena del sur de la provincia de Limón, Costa Rica, pues es una de las más desprotegidas del país, ya que no cuenta con muchos recursos de personal e infraestructura y sus metodologías educativas son un poco obsoletas. En muchos casos, los procesos educativos se enfocan en la memorización y reproducción de textos educativos desactualizados y descontextualizados, con docentes que, en ocasiones, no son personas nativas de la comunidad - quienes se denominan en lengua bribri como sikuas e imparten su clase en español-. La institución de la zona indígena lleva como nombre Centro Educativo Sulayom, ubicado en la comunidad de Amubri, Talamanca, con estudiantes de zonas cercanas como: Amubri, Bambú, Bris, Cachabri, Gavilán, Katsi, Rancho Grande, Shiroles, Sibujú, Surayo, Suretka, Telire, Uatsi y Urén, y con edades entre los dieciséis y los veinticuatro años.

Un elemento llamativo de esta experiencia fue la consideración de la cosmovisión indígena, pues es un factor fundamental para lograr establecer puentes cognitivos entre la materia, la expresión gramatical y su entorno social, sobre todo debido a la carencia de dominio del 
lenguaje bribri del investigador. En contraposición, el estudiantado si habla y se expresa claramente en español.

Las condiciones de acceso y las horas de viaje no fueron impedimento para llegar a la zona. En este territorio, el aprendizaje del lenguaje se desarrolla desde los primeros años, en su lengua y escritura materna y, al llegar a la escuela, se enseña el uso, la lectura y la escritura del español, utilizando métodos de lectoescritura, lo cual propicia que la lengua indígena pase a segundo plano y "con el transitar de los años" se comience a olvidar.

\section{Educación "bulímica" y "anestesiante", un problema actual en Costa Rica}

La docencia debería centrarse en los retos y problemas que acarrea el mundo actual, también se deberían plantear algunas preguntas acerca de: ¿Cómo estudian la juventud en la actualidad? ¿Cómo mejorar los procesos de aprendizaje? No obstante, se apunta hacia otro rumbo: la memorización y fragmentación de contenidos. Por ende, el día del examen el estudiantado se sienta ante las hojas, regurgita o expulsa los conocimientos que se tragó apresuradamente teniendo como consecuencia que uno o dos días después no recuerde nada de lo que vio en clase ni lo que estudió para las pruebas.

El anterior fenómeno da pie a realizar otras preguntas: ¿Cómo son las clases que se están dando en los centros educativos? ¿Cómo aprende la población estudiantil? Ambas interrogantes llevan, ineludiblemente, al tema de la metodología de trabajo, donde la memorización y la repetición campean.

Lo cierto del caso, con algunas excepciones, es que este tipo de enseñanzas y forma de planear las clases son completamente "anestesiantes", en las cuales el estudiantado acude, se sienta en filas, sin poder hablar, debe poner atención a su docente, quien posee el conocimiento y quien le como tabula raza -adultocentrismo- para que al final una alarma le indique la salida o la entrada a clases -condicionamiento clásico-.

Entonces, ¿dónde quedan los espacios de socialización?, ¿dónde quedaron las discusiones propositivas?, ¿será que el personal docente, al entrar a un salón de clase, se abstrae de todo tipo de sentimientos, motivación, sensibilidad, amor por su trabajo y por sus estudiantes? 
Las interrogantes anteriores son analizadas por D'Antoni, Gómez, Gómez y Soto (2012), en su libro La escuela en cuestionamiento: Diálogos sobre la resistencia escolar en procesos pedagógicos emergentes. En dicha obra, se señala que la escuela es "una institución moderna, agotada y en crisis, ya que la escuela no educa para la vida 0 , dicho de otro modo, esta desconectada de la vida y del espacio social de los educandos" (p. 25).

Si se analiza la educación costarricense desde visiones y estudios desarrollados por élites, esta parece ser una de las mejores posicionadas en Centroamérica y a nivel de América Latina, pues como se expone en el Cuarto Informe Estado de la Educación (Programa Estado de la Nación, 2013):

El sistema educativo costarricense sigue mostrando avances importantes, en los últimos años hubo mejoras en el financiamiento, se aplicaron las oportunidades de acceso, se renovó parcialmente la oferta educativa, se aprobaron nuevas disposiciones a favor de los estudiantes y se inició un proceso de trasferencia de competencias de gestión en las escuelas y colegios. (p. 27)

Al analizar la educación costarricense desde las instituciones de poder, exponen que en las últimas décadas se han alcanzado elevados niveles de calidad y cobertura, pero si se analiza desde la realidad de aula de los contextos educativos menos favorecidos, lo que se evidencia es el uso de "parches", los cuales brindan soluciones a corto plazo.

Es en este punto en el cual, exponen D'Antoni, Gómez, Gómez y Soto (2012), "actualmente, la escuela se debate en una doble función, por un lado, reproducir el status quo, y, por otro, lado resistir produciendo autonomía para la liberación" (p. 54). El gran dilema es saber ¿quién define los factores críticos que están incidiendo en esta problemática? Se revela, así, el sistema educativo estatal como primer responsable, pues este ve en la educación un adoctrinamiento del cual la juventud sumisa es mejor de gobernar y un pueblo ignorante, es un pueblo que no crítica y no es consciente de su verdadera realidad.

En un mundo donde se ha promovido la comunicación digital, la libre expresión de ideas y la difusión de ideologías contra hegemónicas, es complicado y hasta contradictorio, decir que solo los grupos docentes o los gobiernos manejan la información sin cuestionar. Basándose 
en las ideas contra hegemónicas, Gadotti (2011), en el prólogo del libro, Gramsci y la educación: Pedagogía de la praxis y políticas culturales en América Latina (Hillert, Ouviña, Rigal y Suárez, 2011), reflexiona en torno al hecho de que: "Educar es siempre tomar partido, mostrar una dirección, asumir valores, comprometerse, pero sin adoctrinar o manipular. Educar es concientizar, desfetichizar, desmitificar, tornar visible lo que fue ocultado para oprimir" (p. 8).

\section{Pedagogía teatral y teoría sociocultural, aportes teóricos a la "en- fermedad constante de la memorización y homogeneidad mental"}

La educación se ve cada día más sumergida en un alfabetismo condicionante y opresor, donde las estructuras de poder responden a un sistema neoliberal y sus intereses. Esta condición de opresión exige desarrollar la capacidad de reaccionar de todos los agentes educativos: personal docente, directivo, estudiantes, grupos familiares y comunidad. En este sentido, es imperante, primero, una resistencia al conductismo dominante; luego, acciones más fundamentadas con el fin de reorganizar la sociedad educativa, que activen la crítica, la creatividad, la percepción, la sensibilización de los aprendizajes y, lo más importante, que fomenten una educación para la paz y la vida social. Por ende, serán acciones contrahegemónicas y pretenden la emancipación para construir y transformar, entre todos y todas, un modelo de desarrollo alternativo, humanista, autónomo, sociocultural, inclusivo y utópico que favorezca la enseñanza-aprendizaje de estudiantes en Amubri o en otras comunidades del país.

Por lo cual, es posible que, por medio de un cambio en las metodologías didácticas, el nivel de compromiso y la mediación docente se logre incentivar, en las aulas, el cambio, generando un conocimiento significativo que cale en las mentes y corazones estudiantiles. En este sentido, el teatro constituye una valiosa herramienta didáctica, pues facilita la expresión artística-corporal, además de integrar teorías clave para el mejoramiento de la educación, como lo son el aprendizaje significativo, el contexto sociocultural y la educación crítica.

La pedagogía teatral, como expone García-Huidobro (1996), surgió "en Europa como una respuesta educativa a la necesidad de renovar metodologías que optimizarán el proceso de aprendizaje, profundamente alterado por la segunda Guerra Mundial y sus consecuencias en el orden social, cultural, político y económico" (p. 15). Esta metodología 
"se constituye como un aporte concreto [de acción pedagógica] para apoyar el proceso de transición [mencionado anteriormente], de la concepción conductista imperante hacia una visión" (García-Huidobro, 1996, p. 15) más artística, motora y sensible, logra una gran atención y motivación en los grupos de estudiantes.

La pedagogía teatral, según García-Huidobro (1996), tiene cuatro tendencias importantes desde sus inicios hasta la actualidad: la tendencia neoclásica, liberal, radical y la del socialismo crítico. De acuerdo con dicha autora, el teatro permite reflexionar:

en torno a la necesidad de asumir tanto el interés genuino del alumno de expresar su emotividad, como el rol cultural del teatro en una sociedad... El rol social y crítico articulado por el teatro cuando cumple una función en relación a otro lidera esta tendencia, cuya visión renovadora y actual orienta tanto [al estudiante como al docente]. (p. 16)

El uso del teatro constituye un valioso recurso de aprendizaje que motiva y facilita los procesos de enseñanza y aprendizaje, así como de la capacidad expresiva, fuera de todos los parámetros formales e informales. El arte de actuar o simular situaciones permite estructurar un soporte significativo, para lograr el objetivo meta, volver o trasformar esa educación "bulímica" y "anestesiante" en una educación creativa, contextualizada, critica y propositiva.

La utilización de la pedagogía teatral, como recurso metodológico y didáctico dentro y fuera de las aulas, ayuda al personal docente a introducir temas y hacer más entretenida la materia, lo que contribuye a activar y volver más ameno el trabajo de aula, pues tanto discentes como facilitadores pueden simular acciones, personajes, vivenciar los sucesos del pasado y hasta poder evocar temas que suceden tanto en el presente como pronosticar para el futuro.

Lo anterior permite realizar un enlace con otras teorías educativas que respaldan la significatividad del teatro -también visto como una actividad lúdica- en el proceso de enseñanza y aprendizaje; ejemplo de ello expone Baquero (2009), pues "como se sabe el juego, el aprendizaje en contextos de enseñanza e, incluso, los procesos de trabajo son mencionados por el propio Vigotsky como actividades productivas de desarrollo o, más específicamente, como productoras de ZDP” (p. 9). 
Con respecto a lo anterior, Chaves (2011) sugiere que este "concepto es básico para los procesos de enseñanza y aprendizaje pues la educadora y el educador deben tomar en cuenta el desarrollo del estudiante en sus dos niveles: el real y el potencial para promover niveles de avance y autorregulación" (p. 62). Además de estos dos niveles, se busca llegar a un desarrollo próximo, en el cual el conocimiento y los signos psicológicos emerjan de la interacción sociocultural y de la evolución, como es el lenguaje, la escritura y el cálculo. Por ello, el estudiantado, al entrar en contacto con la cultura a la que pertenece, se apropia de los signos que son de origen social para, posteriormente, internalizarlos.

Port anto, el teatro como juego dramático es un buen recurso educativo que colabora con la significatividad e incluso se puede desarrollar un eje transversal con el aprendizaje sociocultural, ya que sus finalidades de aprendizaje son muy semejantes. Para evidenciar esto Vygotsky (2000), expone el siguiente ejemplo:

Si suponemos que en el caso dado se reactivan conexiones más antiguas que sustentan la nueva memorización, sería lógico confiar que pasaría lo mismo en todas las demás estructuras. Los experimentos, sin embargo, demuestran otra cosa. Demuestran que el niño, en la inmensa mayoría de los casos, llega a crear estructuras completamente nuevas y no restablece las antiguas. Por ejemplo, cuando el niño memoriza la palabra «teatro» con ayuda de un dibujo que representa un cangrejo en la orilla, forma una estructura auxiliar especial: «El cangrejo mira las piedrecillas del fondo, son bonitas y para él es como el teatro.» Si en el primer ejemplo cabe hablar de que el niño revive una historia oída por él muchas veces, en el segundo es indudable que el niño relaciona por primera vez la palabra «cangrejo» y «teatro». Acaba de establecer esa relación en aquel preciso instante para memorizar la palabra «teatro». Desde el punto de vista asociativo sería extremadamente difícil explicar por qué una representación tan compleja se memoriza más fácil que un simple nexo asociativo. (p. 250)

Se podría concluir que la pedagogía teatral aporta una metodología activa que trabaja todo lo relacionado con el mundo afectivo de las personas. Esta, a su vez, propicia el desarrollo de la vocación 
humana de los individuos por sobre la artística, es decir, es una disciplina articulada para todos los sujetos y no solo para los más dotados como futuros artistas. Al comprender la capacidad del juego dramático desarrollada en estudiantes como recurso educativo fundamental y como punto de partida para posteriores indagaciones pedagógicas, se debe entender que el teatro no es un fin en sí mismo, sino un medio al servicio del estudiantado. Por otra parte, el teatro permite respetar la naturaleza y las posibilidades objetivas estudiantiles, según su etapa de desarrollo del juego, estimulando sus intereses, capacidades individuales y colectivas en un clima de libre expresión. Siguiendo el trabajo de García-Huidobro (1996), se dice:

Es sabido que apenas el niño adquiere conciencia de un mundo extraño a él quiere poseerlo, y que al no poder lograr dicho deseo se rebela liberando, a través de la imaginación, la magia y el juego, su carga de frustración. (p. 19)

La cita anterior muestra la importancia de los juegos teatrales y el valor del uso de actividades lúdicas para el desarrollo de la capacidad de creación artística, la compensación imaginativa de sus razonamientos, experiencias y afectos, por ende, la actividad lúdica genera que estudiantes se liberen y reconstruyan aprendizajes más sociales y significativos para sus vidas.

Sobre el tema de la imaginación y la libertad, Freire (2003) indica que: "nadie educa a nadie, nadie se educa solo y los hombres se educan entre sí mediatizados por el mundo" (p. 19). Por lo cual, es necesario potenciar el aporte que realiza el juego dramático a la autonomía del estudiantado. Junto a esto, Freire (2012) señala que "es realmente fundamental qué el hijo asuma ética y responsablemente la decisión fundadora de su autonomía. Nadie es autónomo primero para después decidir. La autonomía se va constituyendo en la experiencia de varias, innumerables decisiones, que van siendo tomadas" (p. 101). Por otra parte, Matos, Pineda y Vásquez (2010), exponen:

En este sentido, para la mediación pedagógica y el establecimiento de las zonas mencionadas, se presentan como estrategias innovadoras: la Visión Compartida, la Imaginación Creadora y el Trabajo en Equipo como dinámicas que sustentan las interacciones 
sociales propias del proceso de enseñanza y aprendizaje, simétricas con los pares y asimétricas con los docentes o pares avanzados. (p. 185)

Por las razones anteriores, se puede concluir que la pedagogía teatral no queda aislada de otras teorías, pues está vinculada estrechamente con los postulados de grandes escritores y escritoras y profesionales de la pedagogía contemporánea y del siglo XX. En este sentido, el teatro constituye una gran herramienta para el desarrollo de aprendizajes significativos, en favor de un cambio en el modelo pedagógico de la comunidad, con el fin de hacer frente al imperio del conductismo que gobierna en muchas aulas e incluso centros de formación docente.

\section{Implementación de la pedagogía teatral en la comunidad de Amubri}

La comunidad de Amubri está ubicada a cuatro kilómetros después de la comunidad de Suretka, Talamanca. Para llegar a esta zona hay que cruzar el rio Te líre, en una panga ${ }^{3}$. El colegio cuenta con una infraestructura deseable, tres pabellones de aulas, comedor, biblioteca y un usure (casa cónica). La población estudiantil, o como la nombran D’Antoni, Gómez, Gómez y Soto (2013), coinvestigadores, fue aproximadamente de diecisiete a veintiocho estudiantes, mujeres y hombres entre los dieciséis y los veinticuatro años de edad, que cursaban décimo o undécimo. Las sesiones de trabajo tuvieron una duración de una hora con cuarenta minutos, cada quince días y durante los meses de marzo a setiembre del 2013 en el mismo centro educativo.

Los contenidos abordados fueron de la asignatura de estudios sociales para el nivel de décimo año, entre ellos: geografía mundial; antecedentes, causas y consecuencias de la Primera Guerra Mundial; periodo entre guerras, Guerra Fría y los conflictos étnicos y religiosos del mundo.

Dentro de los recursos utilizados se encuentran: atuendos, ropa como sacos, camisas bufandas, gorros, sombreros, palos, hilos, guantes, anteojos, papeles periódicos y los recursos de aula, como los pupitres, sillas, mesas, pizarras, entre otros. La implementación se dio en todas las sesiones-tutorías, en las cuales el facilitador promovió el trabajo y autonomía de su equipo coinvestigador; esto, con el fin de, primero,

3 Pequeña embarcación de fondo aplanado, recomendada para la navegación en ríos poco profundos. 
generar un ambiente de confianza en el aula y, segundo, familiarizar al estudiantado a un ambiente teatral. De esta manera, a partir del empleo de una metodología práctica y activa, se dan ciertas pautas metodológicas para que, posteriormente, en las dos últimas sesiones y con un ambiente de confianza ya construido, el estudiantado se empodere del espacio áulico y desarrolle los contenidos facilitados por su docente de manera artística.

La evaluación y los resultados de la investigación no fueron analizados cuantitativamente, por ende, no se dio el uso de notas numéricas; sin embargo, en cada sesión se brindaba a cada estudiante un instrumento evaluativo de corte cualitativo en el cual se denotaban los avances o retrocesos. El instrumento estaba elaborado con preguntas abiertas sobre las temáticas abordadas: ¿qué se aprendió de los contenidos?, ¿cómo fue el desenvolvimiento del personal docente facilitador?, ¿cuál fue la percepción sobre la clase? y ¿cómo estudiante, ¿qué más aportaría para que el proceso de enseñanza y aprendizaje fuera más significativo?

Como se mencionó anteriormente, una de las demandas que exigen los estudiantes en la actualidad, es un sujeto mediador que pueda brindar una explicación de la materia, que pueda hacer conexiones de esta con la actualidad y que valore su posición humana ante la juventud, por ende, su explicación tiene que estar en su mismo lenguaje. En este aspecto, la implementación dio luces de progreso en cada sesión, pues el estudiantado argumentaba que la estrategia metodológica era muy diferente a la que el grupo estaba acostumbrado en sus colegios. En sus propias palabras lo ejemplifican ${ }^{4}$ : "las clases del colegio son más de recibir materias y un poco de explicación de cada tema y los de acá son más de explicación..." (Encuesta \# 1, María, 2013). Otro estudiante comenta, "un poco nada más que el profe de Estudios, resume y busca dar a los estudiantes una idea central sobre el tema". El profesor que nos da clase es muy seria y rápida" (Encuesta \# 1, Juan, 2013). Una estudiante expone "Nelson explica leído y de una forma muy dura de entender. Arturo Forma excelente, todo entendido" "No es semejante tiene cierta variedad el tutor para la enseñanza y hace la clase más dinámica en contraste a las de los centros educativos" (Encuesta \# 1, Yarixza, 2013).

4 Los nombres de estudiantes son ficticios para mantener el anonimato, la trascripción es literal y tomada de las encuestas, por ende, se toma en consideración la ortografia y la redacción originales. 
También dieron su opinión sobre la metodología desarrollada y se expone "muy bien e interesante ya que así he entendido mejor y me parece importantísimo que el siga con su estrategia y su forma de explicar" (Encuesta \# 2, Juan, 2013). Otro estudiante mencionó: "Muy bien dinámico, particular a otros en el tema visto excelentes gestos, palabras entendibles anima la clase explica los importantes temas, es activo" (Encuesta \# 2, Gardel, 2013). Se indica por parte de un estudiante: "Esta súper chiva, estudios es historia y dramatizado por eso es aún más fácil de entender, la estrategia utilizada es muy dinámica y me parece bien, así siento que la materia es muy divertida y entiendo más rápido" (Encuesta \# 3, Tommy, 2013). Una estudiante menciona "Excelente es muy explicativo y dinámico en lo personal la metodología que utilizo el profesor fue muy interesante porque utilizo ejemplos reales para identificarse el estudiante con el tema que en el momento se esté estudiando, entonces se entiende mejor" (Encuesta \# 3, Ruth, 2013). Además, un estudiante expone "Muy excelente, describe todo tal y como tiene que ser es muy abierto al criterio de los estudiantes, pausa la clase si no está bien entendida su materia, no he conocido otro profe que conoce de lo que habla" (Encuesta \# 3, Stewart, 2013). Por último, un estudiante menciona: "Me parece una buena metodología ya que la clase fue muy espontánea y las ilustraciones que el docente hizo se le quedan en la mente a uno" (Encuesta \# 2, Bernal, 2013).

Como se observa anteriormente, el teatro y el juego dramático como herramienta metodológica fueron del agrado de la mayoría de estudiantes; sin embargo, respetando las diferentes posiciones dentro de un ambiente diverso y democrático, un estudiante aporta lo siguiente: "Mucho movimiento, con poca estrategia no fue contundente no se entendía deseo que se esfuerce más" (Encuesta \# 2, Eliecer, 2013). Por ende, se pudo percibir que había la necesidad de adecuar más la metodología, para que este aporte brindado por el coinvestigador no se siguiera dando en las posteriores clases, con ello este fue el único comentario negativo, pues en otras sesiones, el mismo estudiante expreso su interés y hasta participó en el juego dramático en las últimas sesiones.

Esto es muy importante para tomar en consideración, pues uno de los propósitos de la investigación es que se desarrolle una construcción de los aprendizajes de forma dialógica en donde el estudiantado aprenda de los contenidos y el sujeto formador aprenda de las características particulares de sus estudiantes, por ende, que sea un crecer de las dos 
partes involucradas en el proceso de enseñanza y aprendizaje. Por esta razón, el estudiantado brindó varios aportes, recomendaciones y aspectos por mejorar en la metodología empleada: "Creo que sería mejor hacer las clases con demostraciones del docente para que uno pueda recordar más fácil a la hora del examen" (Encuesta \# 4, Eylin, 2013). Un estudiante aportó: "practicas, con exámenes" (Encuesta \# 4, Alex, 2013). Se considera también "película, drama, recreación" (Encuesta \# 4, Igna, 2013). Otro estudiante indicó: "participación de los receptores, películas, powerpoint, juegos, recreación" (Encuesta \# 4, Dimar, 2013). Por ultimo un estudiante expuso: "podría ser videos o carteles, dibujos" (Encuesta \# 1, Justin, 2013).

Evaluar una metodología como el teatro con una evaluación cuantitativa, sumativa no tendría sentido, pues ¿cómo se cuantifica o se da una nota numérica a la creatividad o al esfuerzo? En este sentido, el uso de rúbricas y el aprendizaje por proyectos resultaron más significativos. Por este motivo, durante la aplicación del uso de teatro en la institución seleccionada, se utilizaron preguntas generadoras y se promovió la discusión con el equipo coinvestigador. De este modo, se lograron evidenciar, de una mejor manera, los aprendizajes desarrollados a lo largo de las sesiones de trabajo.

Para citar algunos ejemplos de las sesiones, se les pregunto lo más relevante de la clase anterior a lo que respondieron la "crisis del 29, caída de la bolsa "wallstreet", segunda guerra mundial guerra fría URSS vrs. capitalismo" (Encuesta \# 3, Tommy, 2013). También "repasamos la primera y segunda guerra mundial, sus causas, las consecuencias, la creación del ONU y entramos a la guerra fría, muerte de archiduque, triple entente y triple alianza" (Encuesta \# 2, Eylin, 2013). Otro estudiante indica: "se estudió temas de suma importancia como aclarar conceptos tales como totalitarismo I guerra mundial, crisis económica de 1929 New Deal entre otros" (Encuesta \# 3, Eliecer, 2013). Se expresa por parte de una estudiante: "El final de la I guerra mundial y el inicio de la 2 guerra mundial y que fueron las causas y las consecuencias de estas guerras. Para la siguiente lección íbamos a ver el papel de Japón" (Encuesta \# 3, Ruth, 2013).

Estas breves opiniones dieron una luz de que el trabajo realizado rindió frutos, pues tanto la metodología como los conocimientos actuados y explicados calaron en el grupo de estudiantes y lograron no solo 
apropiarse de estos a nivel personal, sino también cuestionarse cómo en la actualidad se manifiestan el capitalismo y el comunismo.

Para finalizar el proceso de implementación de la metodología, se trabajó el tema de los conflictos étnicos y religiosos, en los cuales el grupo de estudiantes de dividió en subgrupos y se seleccionó un conflicto. Al respecto, debían analizar y después realizar una puesta en escena para el resto de los compañeros y las compañeras. Se asignaron los contextos en donde existen problemas y a un grupo se le consignó el rol de la ONU, en el cual debían proponer soluciones a los conflictos. La actividad se desarrolló con gran entusiasmo y los resultados en el uso del espacio, el contenido de la temática y la expresión corporal fueron muy buenos, además de la creación de propuestas en pro de algunas soluciones a estos conflictos. Se pudo observar en los rostros del equipo coinvestigador, en sus movimientos y en su oralidad, que estaban realizando algo que tal vez solo en juego habían realizado y no como una actividad educativa y potencialmente significativa.

\section{Conclusiones}

En Costa Rica, existe una "maquina domesticadora" que durante años ha realizado acciones en contra de una educación de calidad; esto, para favorecer a los grupos poderosos. Sin embargo, no es la única culpable, pues el "capitalismo salvaje" limita los procesos educativos para que se fomente la mano de obra y las necesidades del mercado. Además, impera un gran estado de confort en el que gran cantidad de docentes han caído, así como los bajos estándares de calidad en su formación y capacitación. Empero, no todo está perdido, hay personal educativo que se levanta día tras día con el afán o convicción de aportar a la educación con nuevas e interesantes ideas, como un día mediante una gran idea y una metodología alternativa se desarrolló en esta investigación.

Los aprendizajes significativos se logran con trabajo y con una buena mediación pedagógica, por ende, el trabajo sociocultural desarrollado como teoría educativa en las clases y el recurso del teatro como implementación en la mediación resultaron de manera cabal para el estudiantado. Este vio una nueva forma de comprender contenidos y en el proceso divertirse y aprenderlo para aplicarlo en un futuro en otras materias académicas o en actividades comunales.

La pedagogía teatral no es ni una receta ni un remedio -ni pretende serlo- para curar las "patologías educativas" en Costa Rica, más 
bien incentiva a otras manifestaciones para que se liberen y generen cambios significativos y sociales en los espacios educativos.

Hay puntos para mejorar en la aplicación de la metodología, pues durante las sesiones de trabajo, hubo altos y bajos, debido a que cada estudiante tiene su manera de aprender, esto obedece a las distintas formas de aprendizaje, por ende, hay que visualizar cómo se puede modificar y moldear la metodología, además de siempre utilizar ejemplos y recursos contextualizados a la zona en donde se esté trabajando, intentar hablar su mismo idioma e insertarse en lo que se pueda con su cultura, solo así se logra la inclusión de la totalidad de estudiantes, la razón de ser de la educación.

Por último, se quiere sensibilizar al pensar en la función docente actual y hacer la siguiente reflexión: En las manos de un doctor o doctora está una vida, de sus acciones depende si vive o muere; pero quien educa tiene en sus manos más de una vida, si las salva, excelente, si mueren, provoca un genocidio educativo.

\section{Referencias}

Baquero, R. (2009). Zona de desarrollo próximo, sujeto y situación: El problema de las unidades de análisis en psicología educativa. Revista Actualidades Investigativas en Educación, 9(número especial), 1-25. doi: 10.15517/AIE.V9I4.9514

Camargo, M., Calvo, G., Franco, M. C., Vergara, M., Londoño, S., Zapata, F. y Garavito, C. (2004). Las necesidades de formación permanente del docente. Educación y Educadores, 7, 79-112. Recuperado de https://educacionyeducadores.unisabana.edu.co/index. php/eye/article/view/550/643

Chaves, A. L. (2011). Implicaciones educativas de la teoría sociocultural de Vygotsky. Revista Educación, 25(2), 59-65. doi: 10.15517/ REVEDU.V25I2.3581

D’Antoni, M. Gómez, J., Gómez, L. y Soto, J. F. (2012). La escuela en cuestionamiento: Diálogos sobre la resistencia escolar en procesos pedagógicos emergentes. San José: Editorial Arlekin.

Freire, P. (2003). El grito manso. Buenos Aires: Siglo XXI Editores.

Freire, P. (2012). Pedagogía de la autonomía: Saberes necesarios para la práctica educativa. Buenos Aires: Siglo Veintiuno. 
García-Huidobro, V. (1996). Manual de pedagogía teatral. Santiago: Editorial Los Andes. Recuperado de http://www.memoriachilena.gob.cl/archivos2/pdfs/MC0050907.pdf

Hillert, F., Ouviña, H., Rigal, L. y Suárez, D. (2011). Gramsci y la educación: Pedagogía de la praxis y políticas culturales en América Latina (Prólogo de Moacir Gadotti). Buenos Aires: Noveduc.

Matos, R., Pineda, Y. y Vásquez, A. (2010). El aprendizaje del arte. Un modelo de mediación basado en la interacción sociocultural. Revista de Investigación, 69, 179-207. Recuperado de https://www. redalyc.org/pdf/3761/376140384008.pdf

Programa Estado de la Nación. (2013). Cuarto Informe Estado de la Educación. San José: Editorama. Recuperado de http://repositorio.conare.ac.cr/bitstream/handle/20.500.12337/672/ Estado\%20de \% 201a\%20 Educaci \%c 3\%b3n \% 204 . pdf? sequence $=22 \&$ isAllowed $=\mathrm{y}$

Vygotsky, L. (2000). Obras escogidas III: Historia del desarrollo de las funciones psíquicas superiores 1931. Madrid: Visor Dis. 
